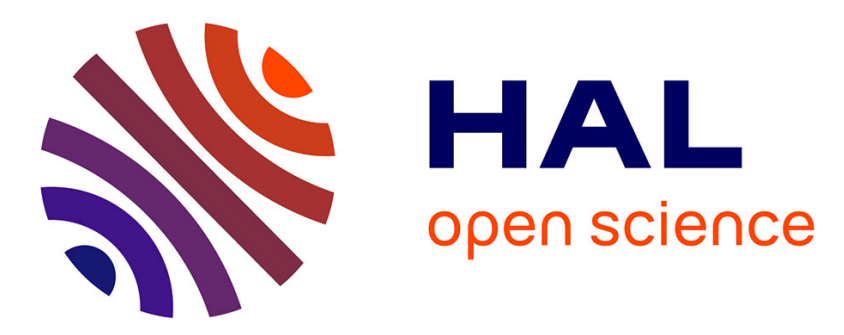

\title{
Comparison of two techniques for the surface analysis of alumina (Al2O3): Inverse Gas Chromatography at Finite Concentration (IGC-FC) and Dynamic Vapor Sorption (DVS)
}

C. Tisserand, Rachel Calvet, S. Patry, Laurence Galet, John A. Dodds

\section{To cite this version:}

C. Tisserand, Rachel Calvet, S. Patry, Laurence Galet, John A. Dodds. Comparison of two techniques for the surface analysis of alumina (Al2O3): Inverse Gas Chromatography at Finite Concentration (IGC-FC) and Dynamic Vapor Sorption (DVS). Powder Technology, 2009, 190 (1-2, SI), pp.53-58. 10.1016/j.powtec.2008.04.058 . hal-01593336

\section{HAL Id: hal-01593336 https://hal.science/hal-01593336}

Submitted on 26 Apr 2019

HAL is a multi-disciplinary open access archive for the deposit and dissemination of scientific research documents, whether they are published or not. The documents may come from teaching and research institutions in France or abroad, or from public or private research centers.
L'archive ouverte pluridisciplinaire HAL, est destinée au dépôt et à la diffusion de documents scientifiques de niveau recherche, publiés ou non, émanant des établissements d'enseignement et de recherche français ou étrangers, des laboratoires publics ou privés. 


\title{
Comparison of two techniques for the surface analysis of alumina $\left(\mathrm{Al}_{2} \mathrm{O}_{3}\right)$ : Inverse Gas Chromatography at Finite Concentration (IGC-FC) and Dynamic Vapor Sorption (DVS)
}

\author{
C. Tisserand, R. Calvet *, S. Patry, L. Galet, J.A. Dodds \\ Ecole des Mines d'Albi-Carmaux, Centre RAPSODEE, UMR CNRS-EMAC 2392, Campus, Jarlard, Route de Teillet, 81013 ALBI CT Cedex 09, France
}

\begin{abstract}
A B S T R A C T
Inverse Gas Chromatography and Dynamic Vapor Sorption are two methods of solid surface characterization isotherms. The exploitation of the adsorption and desorption isotherms leads to the calculation of specific surface area and surface energy of the divided solids under test. The powders used are $\gamma$ and $\alpha$ alumina. The aim of this study is to compare the results obtained by these both techniques.
\end{abstract}

Keywords:

Inverse Gas Chromatography

Dynamic Vapor Sorption

Adsorption and desorption isotherms

Specific surface area

Surface energy

Alumina

\section{Introduction}

This paper deals with two methods of characterization of finely divided solids: Inverse Gas Chromatography at Finite Concentration (IGC-FC) and Dynamic Vapor Sorption (DVS). They give access to physico-chemical properties such as specific surface area, surface energy, BET constant, of the powder surface under test. Both techniques consist in sending a gas stream of water or organic molecules on the surface of the solid. IGC is a method based on chromatographic peak analysis to determine the desorption isotherm of the probe molecule while DVS is a method based on the increase of the solid mass to determine the adsorption and desorption isotherms. The object of the present study is to determine the isotherms of water and different organic molecules and to compare the results obtained by IGC-FC and DVS.

\section{Theory}

\subsection{Inverse Gas Chromatography}

In contrast to conventional gas chromatography, Inverse Gas Chromatography involves the adsorption of a known adsorptive on an unknown adsorbent (solid sample). IGC uses clearly identified molecules, called probes, to determine surface properties of the material packed into the column. The adsorbent is placed in the GC column while the adsorptive is a gas carrying the probe molecules. Two types of IGC may be distinguished: Infinite Dilution (IGC-ID) or Finite solute Concentra-

\footnotetext{
* Corresponding author.

E-mail address: rachel.calvet@enstimac.fr (R. Calvet).
}

tion (IGC-FC) [1]. This paper deals with IGC-FC where all the surface of the solid is covered with the probe molecule, so all the solid surface interacts with the probes, contrary to IGC-ID where only a few molecules are injected and interact mainly with high energy sites. IGC-FC gives access to isotherms of water or different organic molecules and allows calculating specific surface areas, BET constants, and distribution functions of adsorption sites. Two different experimental methods to obtain sorption isotherms may be distinguished: the Elution Characteristic Point method (ECP) and Frontal Analysis.

\subsubsection{Inverse Gas Chromatography with the Elution Characteristic Point method (ECP)}

This method allows the determination of desorption isotherms of organic vapors. It is a simple technique, requiring no special equipment other than a commercial analytical chromatograph, and only one experiment is required to determine each isotherm. In ECP, a large quantity of probe is passed through the column leading to a very deformed chromatographic peak. This deformation is due to the fact that the last molecules leaving the injector arrive at a saturated surface, which decreases their retention time. In the experiment, increasing quantities of probes are injected, and the diffuse fronts of the chromatographic peaks are as shown on Fig. 1. The peak summits appear at lower retention times (chromatograms c1, c2 and c3) until the chromatogram c4 is obtained when there is a monolayer of probe at the surface of the solid. If the amount injected is further increased, we obtain the chromatogram c5, where the plateau shows a multilayer adsorption of the probe.

Fig. 2 presents the evolution of chromatographic peaks obtained with the injection of increasing volumes of probe molecules according to the type of isotherms II or III [1]. The shape of the chromatographic 




Fig. 1. Shapes of the chromatographic peaks for increasing quantities of probe injected.

peak is in relation with the type of isotherm of desorption and here corresponds to an isotherm of type II.

There are always high energy sites at the surface of the solid. When the signal seems to return to the baseline, a small amount of probe molecules can remain fixed irreversibly on the surface of the solid. Thus at the end of the analysis, the temperature of the oven is increased to be higher than the temperature of analysis but lower than the temperature of conditioning. A second peak can then appear corresponding to the thermodesorption of the molecules fixed irreversibly on the surface of the solid at the analysis temperature. In the ECP method the desorption isotherm corresponds to the variation of the desorbed quantity $Q$ at the point of the retention time $t_{\mathrm{r}}$ which is directly related to the net retention volume $V_{\mathrm{I}}$.

$\left(\frac{\partial Q}{\partial c}\right)_{t_{\mathrm{r}}}=\frac{V_{\mathrm{r}}^{\prime}}{m}$

Thus the variation of the quantity desorbed is in relation with the retention volume corresponding to the apparition of a concentration $c$ of the liquid at the exit of the column divided by the mass $m$ of the powder. This equation is only valid if the gas could be considered to be incompressible. However, different parameters such as the shape of the isotherm, the effect of sorption, the compressibility of the gas, the real nature of the gas, the non-ideality of the chromatographic procedure, the thermic effects with the adsorption of the liquid, the variation of the viscosity of the gas with the concentration of the liquid, must also be taken into account as in the following equation due to Conder and Purnell [2].

$\left(\frac{\partial Q}{\partial c}\right)_{t_{\mathrm{r}}}=\frac{V_{\mathrm{r}}^{\prime}}{m\left(1-\alpha \cdot J \cdot y_{0}\right)}$

Here $y_{0}$ is the mole fraction at the exit of the column, $J$ the JamesMartin correction factor involving the compressibility of the gas [3], $\alpha$ a factor for the non-ideality of the vapor of the liquid. We obtain the equation:

$\left(\frac{\partial Q}{\partial c}\right)_{t_{\mathrm{r}}}=\frac{J \cdot D_{\mathrm{s}} \cdot t_{\mathrm{r}}^{\prime}}{m\left(1-\alpha \cdot J \cdot y_{0}\right)}$

with $t_{\mathrm{r}}^{\prime}$ the net retention time and $D_{\mathrm{s}}$ the flow rate of the gas phase at the exit of the column.

The vapor of the liquid is considered to be a perfect gas, so $\alpha=1, J \cdot y_{0}$ becomes negligible in front of 1 if the contribution of the probe to the gas flow rate is less than $5 \%$ of the flow rate in the absence of probe molecules, and the pressure is proportional to the concentration of the liquid. The characteristic equation of IGC-FC is therefore:

$\left(\frac{\partial Q}{\partial P}\right)_{t_{\mathrm{r}}}=\frac{J \cdot D_{\mathrm{s}} \cdot t_{\mathrm{r}}^{\prime}}{m \cdot R \cdot T_{\mathrm{col}}}$

By integration, the desorbed quantity is obtained with the relation:

$Q=\frac{J \cdot D_{\mathrm{s}}}{m \cdot R \cdot T_{\mathrm{col}}} \int_{0}^{P^{\prime}} t_{\mathrm{r}}^{\prime} d P$

The integral $\int_{0}^{P^{\prime}} t_{\mathrm{r}}^{\prime} d P$ of Eq. (5) can be determined from the area $S_{\mathrm{des}}$ under the chromatogram (Fig. 1), the desorbed quantity at $t_{\mathrm{r}}^{\prime}$ is proportional to $S_{\text {des. }}$. The surface $S_{\text {des }}$ can be calculated from the detector coefficient $k_{v}$ which is the proportionality between the mass $m_{\text {probe }}$ of injected probe and the area under the chromatographic peak $S_{\text {peak }}$. The following equation gives this as:

$k_{v}=\frac{m_{\text {probe }}}{S_{\text {peak }}}\left(\frac{g}{\mu V \cdot s}\right)$

The following relation gives also an expression of $k_{v}$ :

$k_{v}=60.10^{6} \frac{V_{\text {inj }} \cdot \rho}{M \cdot D_{\mathrm{s}} \cdot S_{\text {peak }}}\left(\frac{\mu \mathrm{mol}}{\mathrm{cm}^{3} \cdot \mu V}\right)$

with $V_{\text {inj }}$ the volume of probe injected $\left(\mathrm{cm}^{3}\right), \rho$ the density of the probe $\left(\mathrm{g} \cdot \mathrm{cm}^{-3}\right), M$ the molecular mass of the probe $\left(\mathrm{g} \cdot \mathrm{mol}^{-1}\right), D_{\mathrm{s}}$ the
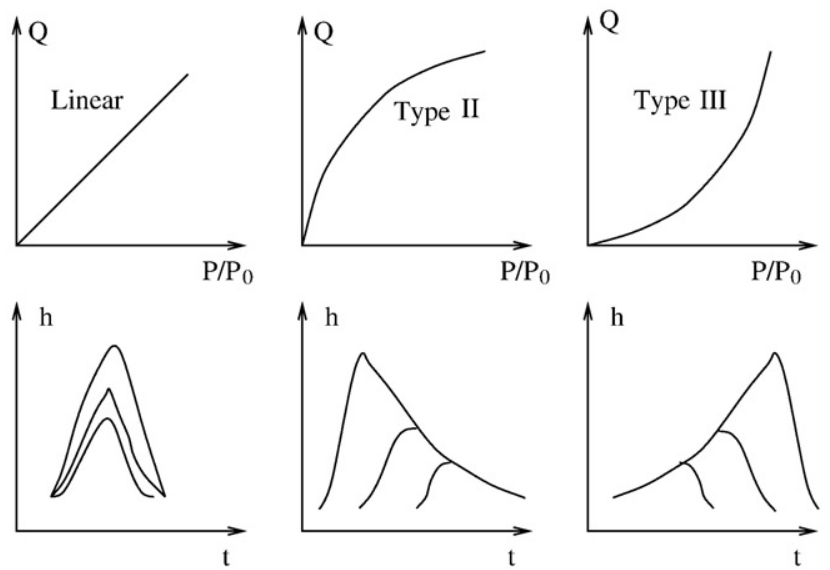

Fig. 2. Sorption isotherms and shapes of the chromatographic peaks. 


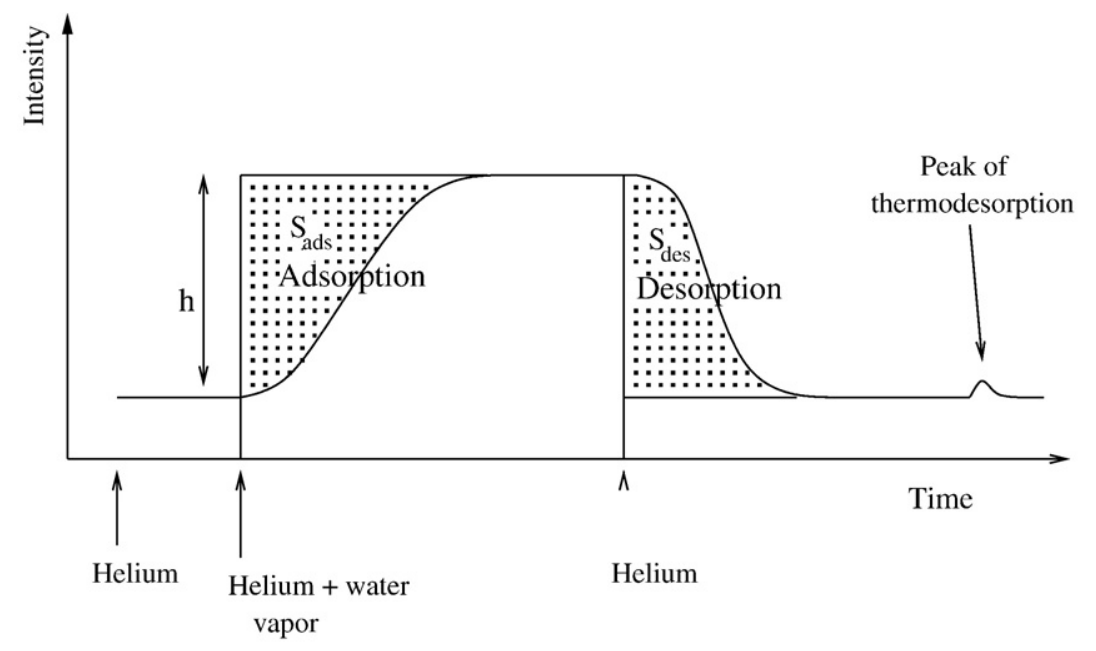

Fig. 3. Chromatogram obtained for one concentration of water in the gas stream in FA.

helium gas flow rate $\left(\mathrm{cm}^{3} \cdot \mathrm{min}^{-1}\right)$ and $S_{\text {peak }}$ the surface of the chromatographic peak associated to the detection of the probe $(\mu \mathrm{V} \cdot \mathrm{s})$.

So this value of the molar volume coefficient $k_{v}$ allows the calculation of the quantity of the probe desorbed at different times along the diffuse front of the chromatographic peak. The quantity desorbed can be related to $S_{\text {des }}$ by the following equation:

$Q=\frac{k_{v} \cdot D_{s}}{m} \cdot D_{\text {des }}$

The coefficient $k_{v}$ is also useful for calculating the molar concentration, and thus the partial pressure of the probe molecule in the gaseous phase from the height $h$ of the chromatographic peak. But the vapor of the probe must be sufficiently diluted to be considered as a perfect gas:

$P=k_{v} \cdot h \cdot R T_{\text {col }}$

where $P$ is the partial pressure of the probe.

\subsubsection{Inverse Gas Chromatography in Frontal Analysis}

This method leads to the determination of an adsorption isotherm. The equipment is a standard chromatograph fitted with a catharometer and a humidity generator which, in the present case, can only deal with water vapor an is not yet compatible with organic vapors.

The column is equilibriated by flowing pure carrier gas (helium) through the column, leading to a stable baseline at the beginning of the experiment. Then the flow is replaced by a continuous stream of carrier gas mixed with water vapor at a constant concentration. The signal increases and when the mass of water adsorbed in the column is constant, it reaches a plateau. Then dry helium is again flowed through the column to bring about the desorption of water. When all the molecules of water are desorbed, and the baseline again is stable the temperature is increased for a thermodesorption phase. The amount of water adsorbed $Q$ in the stationary phase at equilibrium is related to the surface $S_{\text {ads }}$ on the Fig. 3. This amount is calculated from the mass conservation of the probe, considered as a perfect gas, between the entry and the exit of the column [4].

$Q=\frac{D_{\mathrm{s}} \cdot k_{\mathrm{v}} \cdot S_{\mathrm{ads}}}{m}$

The concentration of the probe at the exit of the column is defined using the relation:

$C_{\mathrm{s}}=\frac{P_{\mathrm{sat}}}{R \cdot T_{\mathrm{col}}} \cdot \frac{P_{\mathrm{atm}}}{P_{\mathrm{atm}}+\Delta P}$
Here $P_{\text {sat }}$ is the pressure in the humidity saturator, $R$ the universal gas constant, $T_{\text {col }}$ the temperature in the column, $P_{\text {atm }}$ the atmospheric pressure and $\Delta P$ the pressure drop in the column.

Thus the quantity $Q$ of water adsorbed on the solid contained in the column is given by the relation:

$Q=\frac{1}{m \cdot h} S_{\mathrm{ads}} D_{\mathrm{s}} \frac{P_{\mathrm{atm}}}{P_{\mathrm{atm}}+\Delta P} \frac{P_{\mathrm{sat}}}{R \cdot T_{\mathrm{col}}}$,

If the injected probe is sufficiently diluted, the perfect gas law applies and the relative pressure is obtained from the height of the signal (9).

Knowing the quantity of water adsorbed on the solid contained into the column, gives access to the water adsorption isotherm, by the plot of $Q$ versus relative pressure $P / P_{0}$. Different points of the isotherm can be obtained by varying the water concentration in the gas stream.

With this method, it is also possible to plot the desorption isotherm from the surface $S_{\text {des, }}$, but in this paper, we only work with the adsorption isotherm.

\subsection{DVS}

Dynamic Vapor Sorption (DVS) is a well-established gravimetric method for the determination of vapor sorption isotherms. It consists in measuring the mass of vapor absorbed by a solid sample as a function of the vapor pressure. The sample is placed in a stainless steel mesh sample pan which allows direct vapor flow to all sides of the powder. This sample pan is placed in the DVS instrument at the desired temperature and dried at $0 \%$ relative humidity $(\mathrm{RH})$ to establish the dry mass. After a stable, dry mass is reached, the sample is exposed to the relative humidity according this profile: $0-21 \% \mathrm{RH}$ in $3 \% \mathrm{RH}$ increments. Mass equilibrium is taken to be reached at each humidity stage when the change in the mass of the sample is less than $5 \mu \mathrm{g}$ over $10 \mathrm{~min}$. Complete adsorption and desorption isotherms are determined for each powder.

\subsection{Calculation of specific surface area and surface energy}

The isotherms obtained in IGC-FC and DVS can be used to calculate the specific surface area of the powders. The BET model can be used for the isotherms determined by DVS. However, this model cannot be used for the isotherms determined by IGC-FC because on the chromatogram the slope of the desorption front near the maximum is very high, so the number of points is relatively small. As this leads to an instability in the specific surface area calculated by the BET method another method based on the analogy between the BET equation and the Langmuir equation is used. The isotherm is modified by combining the multilayer adsorption correction and polynomial smoothing [5]. 


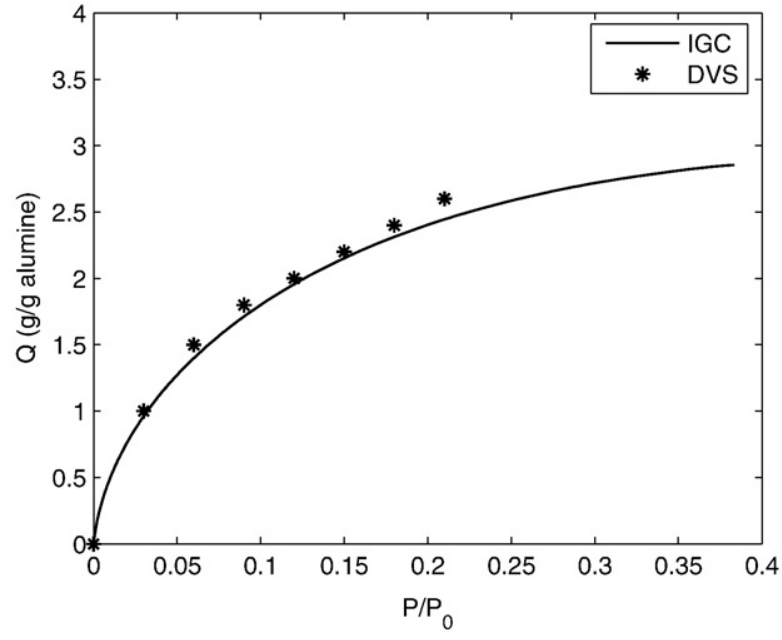

Fig. 4. Sorption isotherms of $\gamma$-alumina obtained in IGC-FC and DVS with the probe octane.

The isotherms also allow the determination of the spreading pressure $\pi_{\mathrm{e}}[6-9] . \pi_{\mathrm{e}}$ is defined as the net change in energy of a surface due to the adsorption of a vapor on the surface. It is determined from the Gibbs equation:

$\mathrm{d} \pi_{\mathrm{e}}=\frac{R T_{\mathrm{col}}}{M S} \cdot Q \cdot \mathrm{d} \ln P$

Here $M$ is the molecular mass of the probe, $S$ the specific surface area, $Q$ the adsorbed quantity (gram per gram of powder) and $P$ the partial pressure of the probe.

To calculate the value of $\pi_{\mathrm{e}}$ corresponding to the adsorbed quantity $Q$ at an equilibrium pressure $P$, Eq. (13) has to be integrated between $\varepsilon$ and $P^{\prime}$ with $\varepsilon$ strictly positive and closed to zero:

$\pi_{\mathrm{e}}=\frac{R T}{M S} \int_{\epsilon}^{P^{\prime}} Q \cdot \mathrm{d} \ln P$

This equation can be transformed and gives this expression:

$\pi_{\mathrm{e}}=\frac{R T}{M S} \int_{\epsilon}^{P^{\prime}} \frac{Q}{P} \cdot \mathrm{d} P$

In this investigation, we calculated the spreading pressure between $\varepsilon$ and the pressure obtained when a monolayer of probe is created at the surface of the solid. Indeed the BET model assumes that the adsorption energy of the first layer is equal to the adsorption energy of the second layer and this latter is equal to the liquefaction energy of the probe, so we consider the adsorption energy at the monolayer.

Fig. 4 shows two isotherms obtained for the $\gamma$-alumina in IGC-FC and DVS.

Fig. 5 gives the slope of $\frac{Q}{P}$ against $P$. It allows the calculation of $\pi_{\mathrm{e}}$ from the area under the curve.

The work of adhesion $\left(W_{s-1}\right)$ and the surface energy $\left(\gamma_{s}\right)$ of the solid can be calculated from the equilibrium spreading pressure $\pi_{\mathrm{e}}$. The interaction between the probe and the solid surface is described by the Young-Dupre equation. This equation can be used with vapor because, after adsorption of the probe on the surface, there is condensation and the behaviour of the probe is the same as a liquid.

$W_{s-1}=\gamma_{l}(1+\cos \theta)+\pi_{\mathrm{e}}$

where $W_{s-1}$ is the work of adhesion, $\gamma_{1}$ the surface tension of the liquid probe, $\theta$ the contact angle between a droplet of liquid sitting on the surface.

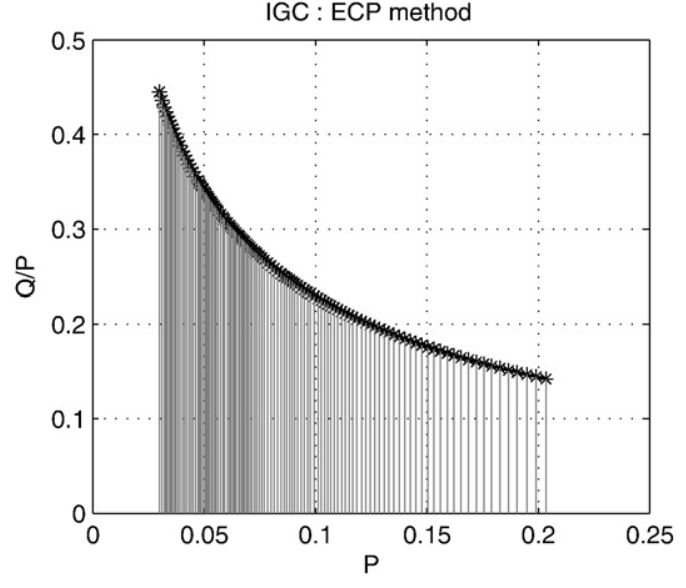

Fig. 5. Slope of $Q / P$ against $P$ for the $\gamma$-alumina obtained in IGC-FC with the probe octane.

With a well wetting liquid, the contact angle becomes equal to zero and the previous equation simplifies to the following one:

$W_{s-1}=2 \gamma_{1}+\pi_{e}$

The equation of Owens and Wendt [10,11] also gives an expression for the work of adhesion which is useful for calculating the surface energy of the solid knowing the value of the work of adhesion from the previous equation:

$W_{s-1}=2 \sqrt{\gamma_{s}^{\mathrm{d}} \cdot \gamma_{1}^{\mathrm{d}}}+2 \sqrt{\gamma_{\mathrm{s}}^{\mathrm{p}} \cdot \gamma_{1}^{\mathrm{p}}}$

With an apolar probe (octane), the expression (18) of the work of adhesion is simplified because the second part of the equation is equal to zero (the polar component of the octane is equal to zero), $\gamma_{1}^{\mathrm{d}}$ is known, so it is easy to calculate $\gamma_{s}^{\mathrm{d}}$.

A polar probe (toluene or water) allows the calculation of the $\gamma_{s}^{\mathrm{p}}$.

The surface energy of the solid is determined by the sum of the two components.

$\gamma_{\mathrm{s}}=\gamma_{\mathrm{s}}^{\mathrm{d}}+\gamma_{\mathrm{s}}^{\mathrm{p}}$

\section{Materials and methods}

\subsection{Materials}

Alumina powders were supplied by the Baïkowski Company. The two alumina have different crystallographic forms, $\gamma$-alumina which is obtained by thermal decomposition of alum, and the $\alpha$-alumina is obtained by calcination of the $\gamma$-alumina.

The liquids used were octane (linear alkane), toluene supplied by Aldrich and purified water (polar liquids). Table 1 gives the values of the dispersive and polar components of the surface tension of the liquids.

\subsection{Experimental IGC conditions}

\subsubsection{IGC-FA}

The IGC-FA chromatographic experiments are carried out with a HP 5890 Series II (Hewlett Packard) equipped with a catharometer. Helium

Table 1

Surface tension of different liquids $\left(\mathrm{mJ} \cdot \mathrm{m}^{-2}\right)$ at ambient temperature

\begin{tabular}{llll}
\hline Liquid & Dispersive component $\gamma_{l}^{d}$ & Polar component $\gamma_{l}^{p}$ & Overall surface tension $\gamma_{l}$ \\
\hline Octane & 21.8 & 0 & 21.8 \\
Toluene & 26.1 & 2.3 & 28.4 \\
Water & 21.8 & 51 & 72.8
\end{tabular}


Table 2

Values of contact angles with toluene and water on alumina

\begin{tabular}{lll}
\hline Contact angle $\theta$ & $\gamma$-alumina & $\alpha$-alumina \\
\hline$\theta$ toluene & 21 & 18 \\
$\theta$ water & 10 & 16 \\
\hline
\end{tabular}

is used as carrier gas with a flow rate of about $60 \mathrm{~cm}^{3} \cdot \mathrm{min}^{-1}$. The detector temperature is set at $200{ }^{\circ} \mathrm{C}$. The columns are conditioned at $200{ }^{\circ} \mathrm{C}$ overnight, the analyses made at $40{ }^{\circ} \mathrm{C}$ and the thermodesorption at $120^{\circ} \mathrm{C}$. A system has been built to provide a controlled humidity carrier gas to the chromatograph which can send dry or wet helium through the column. The input carrier gas is split into two streams, one of these is kept dry and the other is saturated by passing through a gas bubbler. The humidity of the carrier gas is varied by the changing the mixing ratio of dry to wet gases as fixed by the two mass flow meters controlled by a capacitance probe. A thermostated water bath is used to avoid variations of the temperature of the bubbler and of the saturated vapor pressure of water [12]. The experimental apparatus can supply the column alternatively with a dry or wet carrier gas.

\subsubsection{IGC-FC}

The IGC-FC chromatographic experiments are carried out with a HP 6890 Series (Hewlett Packard) equipped with two flame ionisation detectors (FID). Helium is used as carrier gas with a flow rate of about $30 \mathrm{~cm}^{3} \cdot \mathrm{min}^{-1}$ depending on the experiments. The injector and detector temperatures are set at $250^{\circ} \mathrm{C}$. The columns are conditioned at $200{ }^{\circ} \mathrm{C}$ overnight and the analyses were made at $35{ }^{\circ} \mathrm{C}$. The chromatograms are treated with a software created by Henri Balard (Laboratoire de Chimie Physique, ENSISA, Mulhouse) [13]. Each experiment is repeated three times in order to be sure of the reproductibility of the injection.

\subsection{Measurements in DVS}

The Dynamic Vapor Sorption apparatus (DVS, Surface Measurement Systems) consists in a Cahn microbalance housed inside a temperaturecontrolled cabinet. All experiments are performed at $35^{\circ} \mathrm{C}$. Dry nitrogen is bubbled through the probe to give $100 \%$ relative pressure of the solvent. The relative pressure of the probe is controlled by a computer program which sets the appropriate flows of the wet (100\% relative pressure of probe) and dry sides (dry nitrogen, gas flow rate of $4 \mathrm{~cm}^{3} \cdot \mathrm{min}^{-1}$ ). Some DVS measurements were made on alumina conditioned with the protocol of IGC, the results obtained were the same as with the powders nonconditioned.

\subsection{Measurements of contact angles by the sessile drop method}

Alumina compacts were prepared by pressing $2 \mathrm{~g}$ of powder in an uniaxial press (Instron 5567) at a pressure of $5 \mathrm{MPa}$ for $5 \mathrm{~mm} \cdot \mathrm{min}^{-1}$ for the $\gamma$-alumina and $10 \mathrm{MPa}$ for $5 \mathrm{~mm} \cdot \mathrm{min}^{-1}$ for the $\alpha$-alumina at room temperature. Measurements of contact angles at the surface of the compacts were carried out with a ILMS apparatus (GBX). A water drop $(10 \mu \mathrm{l})$ or toluene drop $(5 \mu \mathrm{l})$ is deposited on the surface of the compacts

Table 3

Specific surface areas $\left(\mathrm{m}^{2} \cdot \mathrm{g}^{-1}\right)$ of alumina measured with argon, octane, toluene and water

\begin{tabular}{|c|c|c|c|c|c|c|c|}
\hline \multirow{2}{*}{$\frac{\text { Probe }}{\text { Technique }}$} & \multirow[t]{2}{*}{ Argon } & \multicolumn{2}{|c|}{ Octane } & \multicolumn{2}{|c|}{ Toluene } & \multicolumn{2}{|c|}{ Water } \\
\hline & & IGC & DVS & IGC & DVS & IGC & DVS \\
\hline$\overline{\gamma \text {-alumina }}$ & 92 & 80 & 78 & 101 & 80 & 64 & 66 \\
\hline$\alpha$-alumina & 4 & 8 & 6 & 7 & 6 & 4 & 4 \\
\hline
\end{tabular}

Table 4

Spreading pressure $\left(\mathrm{mJ} \cdot \mathrm{m}^{-2}\right)$, work adhesion $\left(\mathrm{mJ} \cdot \mathrm{m}^{-2}\right)$ and dispersive component of the surface energy $\left(\mathrm{mJ} \cdot \mathrm{m}^{-2}\right)$ with ECP method and DVS with octane

\begin{tabular}{llllll}
\hline Technique powder & IGC & & DVS & \\
\cline { 2 - 3 } & $\gamma$-alumina & $\alpha$-alumina & & $\gamma$-alumina & $\alpha$-alumina \\
\hline$\pi_{\mathrm{e}}$ & $7 \pm 1$ & $6 \pm 1$ & & $6 \pm 1$ & $7 \pm 1$ \\
$W_{\mathrm{s}-1}$ & 51 & 50 & & 50 & 51 \\
$\gamma_{\mathrm{s}}^{\mathrm{d}}$ & 29 & 28 & & 28 & 29 \\
\hline
\end{tabular}

and its change as a function of time is recorded. Table 2 shows the values of these angles which are averages of three values.

\section{Results and discussion}

Different probes were used in IGC-FC and DVS, in particular isopropanol was tried as a polar probe, but was found to be inapplicable for use with alumina because of absorption phenomena which were added to the adsorption. The alumina powders have strong interactions with liquid alcohols meaning that for IGC experiments requiring a volatile probe toluene was chosen as the polar probe for this study.

The exploitation of the isotherms leads to the calculation of the specific surface areas of the solids. Table 3 gives the values measured with argon (Micromeritics ASAP 2010 Device), octane, toluene (IGC with ECP) and water (IGC-FA).

For $\gamma$-alumina, whatever the probe (toluene, octane and water), IGC and DVS lead to the same specific surface areas except the value with the toluene in IGC. If we compare the results as a function of the probe, the specific surface areas are the highest with argon and the lowest with water. The probes octane and toluene lead to intermediate values. The specific surface area of the $\gamma$-alumina was also measured with nitrogen leading to a greater value $\left(114 \mathrm{~m}^{2} \cdot \mathrm{g}^{-1}\right)$. The steric encombrance of toluene and octane can limit the access to the solid surface and so underestimate the specific surface area compared to that measured with argon. Water is a hydrophilic probe which has only access to the hydrophilic surface, consequently it also underestimates the surface.

Whatever the probe and the technique, $\alpha$-alumina has specific surface areas lower than those of the $\gamma$-alumina. These differences come from the process for producing these powders. $\alpha$-alumina is obtained by sintering $\gamma$-alumina which makes some of the surface disappear.

In IGC and DVS the apolar probe octane allows the calculation of the dispersive component of the surface energy according to Eq. (18). The work of adhesion is determined by Eq. (17), as octane is a well wetting liquid the contact angle is equal to zero.

Table 4 gives the values of spreading pressure $\left(\pi_{\mathrm{e}}\right)$, work of adhesion $\left(W_{s-1}\right)$ and dispersive component of the surface energy $\left(\gamma_{s}^{\mathrm{d}}\right)$ obtained respectively by IGC-FC and DVS using octane.

The polar probe toluene allows the calculation of the specific component of the surface energy of a solid with IGC and DVS. Eq. (17) leads to the calculation of the work of adhesion after the verification of the contact angle equal or close to zero (Table 2), as toluene like octane, is a well wetting liquid. Knowing the work of adhesion and the dispersive component of the surface energy determined with octane, it is simple to calculate the specific component from Eq. (18).

Table 5

Spreading pressure $\left(\mathrm{mJ} \cdot \mathrm{m}^{-2}\right)$, work adhesion $\left(\mathrm{mJ} \cdot \mathrm{m}^{-2}\right)$ and polar component of surface energy $\left(\mathrm{mJ} \cdot \mathrm{m}^{-2}\right)$ with ECP method and DVS with toluene

\begin{tabular}{llllll}
\hline Technique powder & IGC & & & DVS \\
\cline { 2 - 3 } \cline { 6 - 6 } & $\gamma$-alumina & $\alpha$-alumina & & $\gamma$-alumina & $\alpha$-alumina \\
\hline$\pi_{\mathrm{e}}$ & $9 \pm 1$ & $9 \pm 1$ & & $9 \pm 1$ & $10 \pm 1$ \\
$W_{\mathrm{s}-1}$ & 66 & 66 & & 66 & 67 \\
$\gamma_{\mathrm{s}}^{\mathrm{p}}$ & 12 & 14 & & 14 & 14 \\
\hline
\end{tabular}


Table 6

Spreading pressure $\left(\mathrm{mJ} \cdot \mathrm{m}^{-2}\right)$, work adhesion $\left(\mathrm{mJ} \cdot \mathrm{m}^{-2}\right)$ and polar component of surface energy $\left(\mathrm{mJ} \cdot \mathrm{m}^{-2}\right)$ with ECP method and DVS with water

\begin{tabular}{llllll}
\hline Technique powder & IGC & & & DVS \\
\cline { 2 - 3 } & $\gamma$-alumina & $\alpha$-alumina & & $\gamma$-alumina & $\alpha$-alumina \\
\hline$\pi_{\mathrm{e}}$ & $40 \pm 1$ & $42 \pm 1$ & & $40 \pm 1$ & $40 \pm 1$ \\
$W_{s-1}$ & 186 & 188 & & 186 & 186 \\
$\gamma_{s}^{\mathrm{p}}$ & 89 & 93 & & 91 & 90 \\
\hline
\end{tabular}

Table 5 gives the values of spreading pressure $\left(\pi_{\mathrm{e}}\right)$, work of adhesion $\left(W_{s-1}\right)$ and polar component of the surface energy $\left(\gamma_{\mathrm{s}}^{\mathrm{p}}\right)$ obtained respectively in IGC-FC and DVS with the polar probe toluene.

The results obtained with both methods IGC-FC and DVS on each powder, are very similar, for example comparing $\pi_{\mathrm{e}}, W_{\mathrm{s}-1}, \gamma_{\mathrm{s}}^{\mathrm{d}}$ and $\gamma_{\mathrm{s}}^{\mathrm{p}}$. It may be concluded that both methods of characterization give good values of surface energy of a finely divided solid.

Furthermore the values of $\gamma_{s}^{\mathrm{d}}$ and $\gamma_{\mathrm{s}}^{\mathrm{p}}$ obtained for the two different alumina, are quite similar. It would be interesting to compare the same calculations on other solids to examine the influence of the solid surface. The question is the sensitivity of the model calculation of the surface energy to the surface chemistry.

Water is a probe having a polar component and was also used to calculate the specific component of the surface energy of the two alumina. It is also a well wetting solvent because the contact angle is close to zero (Table 2). Eq. (17) allows the calculation of the work of adhesion from the water isotherm and from the dispersive component of the surface energy measured with octane, Eq. (18) allows the calculation of the $\gamma_{\mathrm{s}}^{\mathrm{p}}$.

Table 6 gives the values of spreading pressure $\left(\pi_{\mathrm{e}}\right)$, work of adhesion $\left(W_{s-1}\right)$ and polar component of the surface energy $\left(\gamma_{s}^{\mathrm{p}}\right)$ obtained respectively in IGC-FC and DVS with the water, on the $\alpha$ and $\gamma$ alumina.

The values of the polar component of the surface energy measured with water are higher than those measured with toluene. A possible explanation is the limitation of the Owens and Wendt model. This model is an extension of Fowkes model which is applied to the dispersive component of the surface energy [14]. It seems to be valid with toluene for which the polar component $\left(\gamma_{1}^{\mathrm{p}}=2,3 \mathrm{~mJ} \cdot \mathrm{m}^{-2}\right)$ is low but not with water for which the polar component is higher $\left(\gamma_{1}^{\mathrm{p}}=51 \mathrm{~mJ} \cdot \mathrm{m}^{-2}\right)$.

\section{Conclusions}

The comparison of results from IGC and DVS leads to the same specific surface areas with a same probe on two alumina powders. Comparing different probes, it appears that octane and toluene underestimate the specific surface compared with that determined using argon, probably due to their steric encombrance. Water, a hydrophilic probe, also underestimates the specific surface as it does not access the hydrophobic part of the powder surface. The two techniques lead also to the same results for the calculations of the dispersive or specific components of the surface energy on the two alumina. Differences are observed between toluene and water. The Owens and Wendt model seems to be a limit for these calculations.

\section{References}

[1] J.R. Conder, C.L. Young, Physicochemical Measurement by Gas Chromatography, Whiley-interscience, 1979.

[2] J.R. Conder, J.H. Purnell, Gas Chromatography at Finite Concentration, Transactions of the Faraday Society 65 (1969) 824-838.

[3] A.T. James, J.P. Martin, Gas-liquid partition chromatography: the separation and micro- estimation of volatile fatty acids from formic acid to dodecanoic acid, Biochemical Journal 50 (1952) 679-690.

[4] F. Gritti, W. Piatkowski, G. Guiochon, Comparison of the adsorption equilibrium of a few low-molecular mass compounds on a monolithic and a packed column in reversed-phase liquid chromatography, Journal of Chromatography A 978 (2002) 81-107.

[5] H. Balard, Estimation of the surface energetic heterogeneity of a solid by inverse gas chromatography, Langmuir 13 (1997) 1260-1269.

[6] N.K. Adam, E.A. Guggenheim, Proceedings of the Royal Society 218 (1933).

[7] D.H. Bangham, R.I. Razouk, Transactions of the Faraday Society 33 (1937) 1459-1463.

[8] Z. Kessaissia, E. Papirer, J.B. Donnet, The surface energy of silicas, grafted with alkyl chains of increasing lengths, as measured by contact angle techniques, Journal of Colloid and Interface Science 82 (1981) 526-533.

[9] A.W. Adamson, Physical Chemistry of Surfaces, Sixth Edn, Wiley Interscience, New York, 1990.

[10] D.K. Owens, R.C. Wendt, Estimation of the surface free energy of polymers, Journal of Applied Polymer Science 13 (1969) 1741-1747.

[11] D.H. Kaelble, Dispersion-polar surface tension properties of organic solid, Journal of Adhesion 2 (1970) 66-81.

[12] S. Comte, R. Calvet, J.A. Dodds, H. Balard, Surface properties of low specific surface powders using Inverse Gas Chromatography, Powder Technology 157 (2005) 39-47.

[13] D. Belaidi-Maafa. Etude par chromatographie inverse des propri'et'es superficielles physico-chimiques de mat'eriaux carbon'es. PhD thesis, Universit'e de Haute Alsace, 2006.

[14] F.M. Fowkes, Attractive forces at interfaces, Industrial and Engineering Chemistry 56 (1964) 40-52. 